the cases and classified in an ordinal scale: indentation (3.6\%), flutter (8.9\%), focal (7.7\%) and full collapse $(67.3 \%)$. Focal to full arterial collapse was observed in the M2 regardless of catheter size and perfusion pressure. The likelihood and severity of collapse was positively correlated to size of aspiration catheter and negatively correlated to arterial size and perfusion pressure. OCT detected collapse of the aortic lumen during aspiration thrombectomy in rabbit.

Conclusion Arterial collapse occurs with current aspiration thrombectomy technologies in validated human brain models and in vivo in the aorta of rabbit.

Disclosures Y. Liu: 2; C; Endovascular Engineering. D. Gebrezgiabhier: None. J. Arturo Larco: None. S. Madhani: None. A. Shahid: None. R. Kadirvel: None. W. Brinjikji: None. D. Kallmes: None. L. Savastano: 4; C; Endovascular Engineering.

\section{0-013 ACUTE CAROTID ARTERY STENTING VERSUS BALLOON ANGIOPLASTY FOR TANDEM OCCLUSIONS: A SYSTEMATIC REVIEW AND META-ANALYSIS}

${ }^{1} \mathrm{C}$ Zevallos, ${ }^{1} \mathrm{M}$ Farooqui, ${ }^{1} \mathrm{D}$ Quispe-Orozco, ${ }^{1} \mathrm{~A}$ Mendez-Ruiz, ${ }^{1} \mathrm{~A}$ Dajles, ${ }^{1} \mathrm{~A}$ Garg, ${ }^{2} \mathrm{M}$ Patterson, ${ }^{2} \mathrm{O}$ Zaidat, ${ }^{1} \mathrm{~S}$ Ortega-Gutierrez*. 'Neurology, University of lowa Hospitals and Clinics, lowa City, IA; ${ }^{2}$ Neurology, Mercy Health, St. Vincent Hospital, Toledo, $\mathrm{OH}$

\subsection{6/neurintsurg-2021-SNIS.13}

Background Despite thrombectomy has become the standard of care for large vessel occlusion strokes, acute endovascular management in tandem occlusions (TO), specially of the cervical internal carotid artery (ICA) lesion, remains uncertain. It widely varies according to clinical and technical considerations and proceduralist's preference. Revascularization procedures include carotid artery stenting (CAS) \pm balloon angioplasty (BA), used as a definitive treatment strategy, performed prior to or following intracranial MT; and acute BA alone, suction aspiration, or MT alone which implicate a deferred treatment with endarterectomy or stenting in the following days or weeks. Each treatment carries potential risks that are taken into consideration when selecting the best treatment method. Recent studies suggest a benefit in functional outcomes and reperfusion rates when CAS and MT are performed emergently, without increasing symptomatic intracranial hemorrhage (sICH) risk. However, all the above-mentioned approaches are used in clinical practice.

Objectives Compare efficacy and safety of acute CAS to BA alone on treating the cervical ICA lesion in TO through an aggregated data meta-analysis of the recent literature. Similarly, explore those outcomes' associations with technique approaches and use of thrombolysis.

Methods We performed a systematic review and meta-analysis to compare functional outcomes (modified Rankin scale [mRS] $0-2$ at 3 months), reperfusion, and sICH and 3-month mortality. We explored the association of first approach (anterograde/retrograde) and use of thrombolysis with those outcomes as well. Two independent reviewers performed the screening, data extraction, and quality assessment. A random effects model was used for analysis.

Results 34 studies including 3014 patients with TO (2482 CAS and $245 \mathrm{BA}$ ) were included in our systematic review; and nine, in the meta-analysis. Acute CAS was associated with higher odds of $m R S \leq 2$ (OR 1.95 (95\% CI 1.24, 3.05) and successful reperfusion (OR 1.89 (95\% CI 1.26, 2.83)) with no differences in mortality or sICH rates. Moreover, retrograde approach was significantly associated with $\mathrm{mRS} \leq 2$ (OR $1.72 \quad(95 \%$ CI 1.05 , 2.83)) and successful reperfusion (OR 3.18 (95\% CI 1.50, 6.74)). No differences were found regarding thrombolysis status. Conclusions CAS and retrograde approach had higher odds of successful reperfusion and good functional outcomes at 3 months than BA and anterograde approach, respectively, in TO patients. However, there is still insufficient data regarding antithrombotic therapy and stent patency that might influence the evaluated outcomes. A randomized controlled trial comparing these techniques with structured antithrombotic regimens and safety outcomes will offer definitive guidance in the optimal management of this complex disease.

Disclosures C. Zevallos: None. M. Farooqui: None. D. Quispe-Orozco: None. A. Mendez-Ruiz: None. A. Dajles: None. A. Garg: None. M. Patterson: None. O. Zaidat: 2; C; Cerenovus, Stryker, Penumbra, Medtronic. S. Ortega-Gutierrez: 2; C; Medtronic, Stryker.

\section{0-014 OUTCOMES OF MECHANICAL THROMBECTOMY IN THE EARLY (<6 H) AND EXTENDED TIME ( $\geq 6$ H) WINDOW SOLELY BASED ON NONCONTRAST CT AND CT ANGIOGRAPHY}

P Hendrix*, D Chaudhary, V Avula, V Abedi, R Zand, A Noto, I Melamed, 0 Goren, C Schirmer, C Griessenauer. Geisinger, Danville, PA

\subsection{6/neurintsurg-2021-SNIS. 14}

Objective Current stroke care recommendations state patient selection for mechanical thrombectomy (MT) in the extended time window demand advanced imaging to determine the stroke core volume and hypoperfusion mismatch, which may not be available at every center. We aimed to determine outcomes in patients selected for MT solely based on non-contrast CT (NCCT) and CT-angiography (CTA) in the early $(<6$ h) and extended $(\geq 6 \mathrm{~h})$ time windows.

Methods Consecutive MTs performed for acute large vessel occlusion (LVO) ischemic (ICA, M1, M2) stroke between 02/ 2016- 08/2020 were retrospectively reviewed. Eligibility for MT was based on patient demographics and NCCT (ASPECTS), and CTA. Propensity score matching (PSM) was performed to compare outcomes between time windows.

Results Out of 417 MTs performed, 337 met inclusion criteria resulting in $205(60.8 \%)$ and $132(39.2 \%)$ cases in the 0-6 and 6-24 hour time windows, respectively. ASPECTS was higher in the early time window (9, IQR 8-10) than the extended time window (9, IQR 7-10; $p=0.005)$. PSM yielded 112 well matched-pairs. Equal rates of TICI $2 \mathrm{~b} / 3$ revascularization and symptomatic intracranial hemorrhage were observed. A favorable functional outcome (mRS 0-2) was numerically more frequent in the early window $(45.5 \%$ vs. $33.9 \%, p=0.091)$. Mortality was more frequent in the early window $(25.9 \%$ vs. $17.0 \%, \mathrm{p}=0.096)$.

Conclusion Withholding mechanical thrombectomy due to lack of advanced imaging may not be justifiable for those patients with favorable CT-ASPECTS irrespective of the time window of presentation. Additional investigation is required to determine the value of advanced imaging in the extended time window. Disclosures P. Hendrix: None. D. Chaudhary: None. V. Avula: None. V. Abedi: None. R. Zand: None. A. Noto: None. I. Melamed: None. O. Goren: None. C. Schirmer: None. C. Griessenauer: None. 